\title{
A MEASUREMENT OF THE POLARIZATION-TEMPERATURE ANGULAR CROSS-POWER SPECTRUM OF THE COSMIC MICROWAVE BACKGROUND FROM THE 2003 FLIGHT OF BOOMERANG
}

\author{
F. Piacentini, ${ }^{1}$ P. A. R. Ade, ${ }^{2}$ J. J. Bock,${ }^{3,4}$ J. R. Bond,${ }^{5}$ J. Borrill, ${ }^{6,7}$ A. Boscaleri, ${ }^{8}$ P. Cabella, ${ }^{9}$ C. R. Contaldi, ${ }^{5,10}$ \\ B. P. Crill,${ }^{11}$ P. de Bernardis, ${ }^{1}$ G. De Gasperis, ${ }^{9}$ A. de Oliveira-Costa, ${ }^{12}$ G. De Troia, ${ }^{1}$ G. di Stefano, ${ }^{13}$ \\ E. Hivon, ${ }^{11}$ A. H. Jaffe, ${ }^{10}$ T. S. Kisner, ${ }^{14,15}$ W. C. Jones, ${ }^{4}$ A. E. Lange, ${ }^{4}$ S. Masi, ${ }^{1}$ P. D. Mauskopf, ${ }^{2}$ \\ C. J. MacTavish, ${ }^{16}$ A. Melchiorri, ${ }^{1,17}$ T. E. Montroy, ${ }^{14}$ P. Natoli, ${ }^{9,18}$ C. B. Netterfield, ${ }^{16}$ \\ E. Pascale, ${ }^{16}$ D. Pogosyan, ${ }^{19}$ G. Polenta, ${ }^{1}$ S. Prunet, ${ }^{20}$ S. Ricciardi, ${ }^{1}$ G. Romeo, ${ }^{13}$ \\ J. E. Ruhl, ${ }^{14}$ P. Santini, ${ }^{1}$ M. Tegmark, ${ }^{12}$ M. Veneziani, ${ }^{1}$ and N. Vittorio, ${ }^{9,18}$ \\ Received 2005 July 21; accepted 2006 February 10
}

\begin{abstract}
We present a measurement of the polarization-temperature angular cross power spectra, $\langle\mathrm{TE}\rangle$ and $\langle\mathrm{TB}\rangle$, of the cosmic microwave background. The result is based on $\sim 200 \mathrm{hr}$ of data from eight polarization-sensitive bolometers operating at $145 \mathrm{GHz}$ during the 2003 flight of BOOMERANG. We detect a significant $\langle\mathrm{TE}\rangle$ correlation in the $l$-range between 50 and 950 with a statistical significance of $>3.5 \sigma$. Contamination by polarized foreground emission and systematic effects are negligible in comparison with statistical uncertainties. The spectrum is consistent with previous detections and with the "concordance model" that assumes adiabatic initial conditions. This is the first measurement of polarization-temperature angular cross-power spectra using bolometric detectors.
\end{abstract}

Subject heading: cosmic microwave background

Online material: color figures

\section{INTRODUCTION}

Since Rees' pioneering work (Rees 1968), polarization of the cosmic microwave background (CMB) has been at the center of several theoretical studies. Detailed numerical predictions have been made in the framework of standard inflationary models with primordial adiabatic and scale-invariant fluctuations (see, e.g., Bond \& Efstathiou 1984; Seljak \& Zaldarriaga 1996). CMB polarization data are highly useful for cosmology since they can shed light, for example, on the process of reionization of the intergalactic medium (see, e.g., Efstathiou 1988; Zaldarriaga 1997), on the amplitude of the inflationary gravity-wave background (Crittenden et al. 1995; Zaldarriaga \& Seljak 1997; Kamionkowski

\footnotetext{
1 Dipartimento di Fisica, Università di Roma La Sapienza, Piazzale Aldo Moro 2, 00185 Rome, Italy.

2 Department of Physics and Astronomy, Cardiff University, Cardiff CF24 3YB, Wales, UK.

3 Jet Propulsion Laboratory, Pasadena, CA 91109.

4 Observational Cosmology, California Institute of Technology, Pasadena, CA 91125.

5 Canadian Institute for Theoretical Astrophysics, University of Toronto, Toronto, ON M5S 3H8, Canada.

6 Computational Research Division, Lawrence Berkeley National Laboratory, Berkeley, CA 94720.

7 Space Sciences Laboratory, University of California, Berkeley, CA 94720.

8 IFAC-CNR, 50127 Florence, Italy.

9 Dipartimento di Fisica, Università di Roma Tor Vergata, 00133 Rome, Italy.

10 Theoretical Physics Group, Imperial College, London 5W7 2BW, UK.

11 IPAC, California Institute of Technology, Pasadena, CA 91125.

12 Department of Physics, Massachusetts Institute of Technology, Cambridge, MA 02139.

13 Istituto Nazionale di Geofisica e Vulcanologia, 00143 Rome, Italy.

14 Physics Department, Case Western Reserve University, Cleveland, OH 44106.

15 Department of Physics, University of California, Santa Barbara, CA 93106.

16 Physics Department, University of Toronto, Toronto, ON M5S 3H8, Canada.

17 INFN, Sezione di Roma 1, 00133 Rome, Italy.

18 INFN, Sezione di Roma 2, 00133 Rome, Italy.

19 Department of Physics, University of Alberta, Edmonton, AB T6G 2J1, Canada.

${ }^{20}$ Institut d'Astrophysique, 75104 Paris, France.
}

et al. 1997), and on the nature of primordial perturbations (see, e.g., Spergel \& Zaldarriaga 1997; Bucher et al. 2001). Moreover, polarization can provide further evidence for coherent acoustic oscillations in the early universe, since in this case peaks in the temperature and polarization power spectra are expected to be $\pi$ out of phase (Kosowsky 1999). Unfortunately, given the small amplitude of the signal, current CMB polarization data, while providing an important confirmation of the standard scenario, are unable to provide useful constraints on the parameters of the model. As first suggested in Coulson et al. (1994), measuring the temperature-polarization cross correlation is easier, since the signal is higher and carries most of the cosmological information present in the polarization data. Previous detections of the temperature-polarization angular cross power spectrum have been obtained by the Wilkinson Microwave Anistropy Probe (WMAP) satellite (Kogut et al. 2003) and by the Degree Angular Scale Interferometer (DASI; Kovac et al. 2002; Leitch et al. 2005) and the Cosmic Background Imager (CBI; Readhead et al. 2004) interferometers.

In this paper we present new observations of the temperaturepolarization cross-power spectrum of the CMB anisotropy obtained by the BOOMERANG experiment flown in 2003 January (B03). Results on the temperature and polarization power spectra alone are presented in two companion papers (Jones et al. 2006; Montroy et al. 2006), the instrument and the analysis pipeline producing the maps of temperature and polarization are described in Masi et al. (2006), and the cosmological parameter extraction is in MacTavish et al. (2006).

In the present paper we follow the notation of Zaldarriaga \& Seljak (1997; but see also Kamionkowski et al. [1997]) in which polarization is expressed as two linear combinations of $\operatorname{spin} \pm 2$ multipole moments that have opposite parities, the so-called $E$ (electric) and $B$ (magnetic) modes. In standard cosmological models, the magnetic-type parity combination does not cross-correlate with temperature or the electric-type parity combination. The cosmological information in the polarization-temperature correlation is therefore present only in the $\langle\mathrm{TE}\rangle$ angular power spectrum. We 
also present constraints on $\langle\mathrm{TB}\rangle$ as a useful check for systematics and foregrounds. Nonzero $\langle\mathrm{TB}\rangle$ may also appear in exotic theories due, for example, to the presence of helical flows in the primordial plasma at the time of recombination (Pogosian et al. 2002).

We have performed the analysis of the B03 data using two completely independent pipelines, based on different procedures for the pointing solution, data cleaning, deconvolution, mapmaking, and noise estimation, and different estimation of the calibration factors, beams, receiver transfer functions, polarization efficiencies, and polarizer angles. One pipeline was developed in Italy (IT), the other in North America (NA). Pipelines details are in Masi et al. (2006) and will be described further in subsequent papers. The most important result from this splitting is the overall agreement, which enhances confidence in the result. A comparison of the result from the two pipelines allows a measure of the sensitivity of the result to details of the analysis.

\section{POWER SPECTRA ESTIMATION}

In the analysis reported here we use data from 8 channels at $145 \mathrm{GHz}$, composed of four pairs of polarization-sensitive bolometers (PSBs), (W1, W2), (X1, X2), (Y1,Y2), and (Z1, Z2), with effective angular resolution of 11.5 (FWHM, including pointing jitter). Performance and characteristics of those devices are in Masi et al. (2006), together with the full description of the instrument and of the temperature and polarization maps that are used for the analysis presented here.

Using PSBs, it is possible to obtain maps of the three Stokes parameters: $I$, which describes fluctuations in the brightness of the radiation, and $Q$ and $U$, which describe the linear polarization. The intensity of the CMB is conveniently described in terms of temperature fluctuations $\Delta T$ of a blackbody with respect to a $2.725 \mathrm{~K}$ blackbody and can be decomposed into spherical harmonics as $\Delta T(\hat{\boldsymbol{n}})=\sum_{l m} a_{l m}^{T} Y_{l m}(\hat{\boldsymbol{n}})$. Similarly, the linear polarization $Q+i U$ is decomposed using the spin-2 weighted basis ${ }_{ \pm 2} Y_{l m}$,

$$
(Q \pm i U)(\hat{\boldsymbol{n}})=\sum_{l m}\left(a_{l m}^{E} \mp i a_{l m}^{B}\right)_{ \pm 2} Y_{l m}(\hat{\boldsymbol{n}})
$$

thus defining the scalar field $E(\hat{\boldsymbol{n}})=\sum_{l m} a_{l m}^{E} Y_{l m}(\hat{\boldsymbol{n}})$ and the pseudoscalar $B(\hat{\boldsymbol{n}})=\sum_{l m} a_{l m}^{B} Y_{l m}(\hat{\boldsymbol{n}})$. In the hypothesis that those quantities are Gaussian distributed and that the early universe is isotropic, the cosmological information is encoded in the standard deviations and correlations of the coefficients:

$$
\langle X Y\rangle=\left\langle a_{l m}^{X *} a_{l^{\prime} m^{\prime}}^{Y}\right\rangle=C_{l}^{X Y} \delta_{l l^{\prime}} \delta_{m m^{\prime}},
$$

where the pairs $\langle X Y\rangle$ can be $\langle\mathrm{TT}\rangle,\langle\mathrm{EE}\rangle,\langle\mathrm{BB}\rangle,\langle\mathrm{TE}\rangle,\langle\mathrm{TB}\rangle$, and $\langle\mathrm{EB}\rangle$. Given the isotropy hypothesis, these power spectra can be estimated by averaging over $m$ at each multipole number $l$.

Both IT and NA power spectra estimation pipelines are based on the MASTER method (Hivon et al. 2002) that computes the pseudo- $a_{l m}$ on a fraction of the sphere defined by the function $W(\hat{\boldsymbol{n}})$ that takes into account weighting and sky coverage. This yields the definition of mode-mode coupling kernels that depend only on the weighting scheme. Using an appropriate $l$-binning, it is possible to solve for the underlying angular power spectra, taking into account the binning, the angular resolution of the instrument, the pixelization of the map, and any filter applied to the time stream. The quantity that is binned is the flattened power spectrum,

$$
\mathcal{C}_{l}=\frac{l(l+1) C_{l}}{2 \pi}
$$

For a set of $n$ bins indexed by $b$, with boundaries $l_{\text {low }}^{(b)}<l_{\text {high }}^{(b)}<$ $l_{\text {low }}^{(b+1)}$, the binning operator is defined as

$$
P_{l}^{b}= \begin{cases}\frac{1}{l_{\text {low }}^{b+1}-l_{\text {low }}^{b}}, & 2 \leq l_{\text {low }}^{(b)} \leq l \leq l_{\text {low }}^{(b+1)} \\ 0, & \text { otherwise }\end{cases}
$$

and the power in each bin (hereafter band power) is

$$
\mathcal{C}_{b}=P_{l}^{b} \mathcal{C}_{l}
$$

The method of Hivon et al. (2002) is based on Monte Carlo simulations of signal-only time streams, from simulations of the CMB sky, and of noise-only time streams, from simulations of the instrument. Both simulated data streams are processed in the same way as the real data. The signal-only time streams are used to define the transfer function that describes the effect of data filtering and partial sky coverage on the power spectra. The noiseonly time streams are used to estimate the noise in the power spectra. The signal simulations are obtained by means of random realization of the CMB sky, in temperature and polarization, given an underlying cosmological model, projected in a time stream according to the BOOMERANG scanning strategy. The noise simulations are obtained in time space by means of random realization of the noise power spectrum, iteratively estimated, taking into account noise correlation between channels as described in Masi et al. (2006).

The covariance matrix $\mathbf{M}_{b b^{\prime}}$, which defines the uncertainties in the $\mathcal{C}_{b}$ determination, is estimated by Monte Carlo simulations of signal plus noise as in Hivon et al. (2002). An approximation of the diagonal part of this matrix is given by

$$
\begin{aligned}
\sigma_{\mathrm{TE}, b}^{2}= & \frac{2}{\left(2 l_{b}+1\right) f_{\mathrm{sky}} \Delta l} \\
& \times\left[\mathcal{C}_{\mathrm{TE}, b}^{2}+\left(\mathcal{C}_{T, b}+\frac{N_{T, b}}{B_{b}^{2}}\right)\left(\mathcal{C}_{E, b}+\frac{N_{T, b}}{B_{b}^{2}}\right)\right],
\end{aligned}
$$

where $f_{\text {sky }}$ is the effective observed fraction of sky; $\mathcal{C}_{T, b}, \mathcal{C}_{E, b}$, and $\mathcal{C}_{\mathrm{TE}, b}$ are the band powers of the temperature, the polarization and the polarization-temperature correlation, respectively; $N_{T, b}$ and $N_{E, b}$ are the band powers of the noise in the temperature map and in the $E$ map, respectively; $B_{b}$ is the spherical harmonic transform of the beam; and $\Delta l$ is the bin width. According to this equation, the error bar on each band power is a combination of sampling variance and statistical noise. Those error bars must be minimized by the choice of observation strategy and weighting scheme that is a compromise of wide coverage and deep integration.

\subsection{Weighting}

As described in Masi et al. (2006), observations during the last flight of BOOMERANG were split into three parts: a deep integration over a region as wide as $0.22 \%$ of the sky, centered at R.A. $=82^{\circ} .5$, decl. $=-45^{\circ}$ (hereafter deep region), a shallow integration over a region covering $1.8 \%$ of the sky (including the deep region) centered in the same coordinates (hereafter shallow region), and daily observations of the Galactic plane, which are not used in the analysis reported here. Wide coverage and deep integration are both important for the quality of the result. The wide coverage of the shallow region is useful to reduce sample variance; the deep integration of the deep region is useful to reduce the statistical noise.

The two pipelines use different methods to combine the data in order to obtain a compromise of sample variance and noise. For 


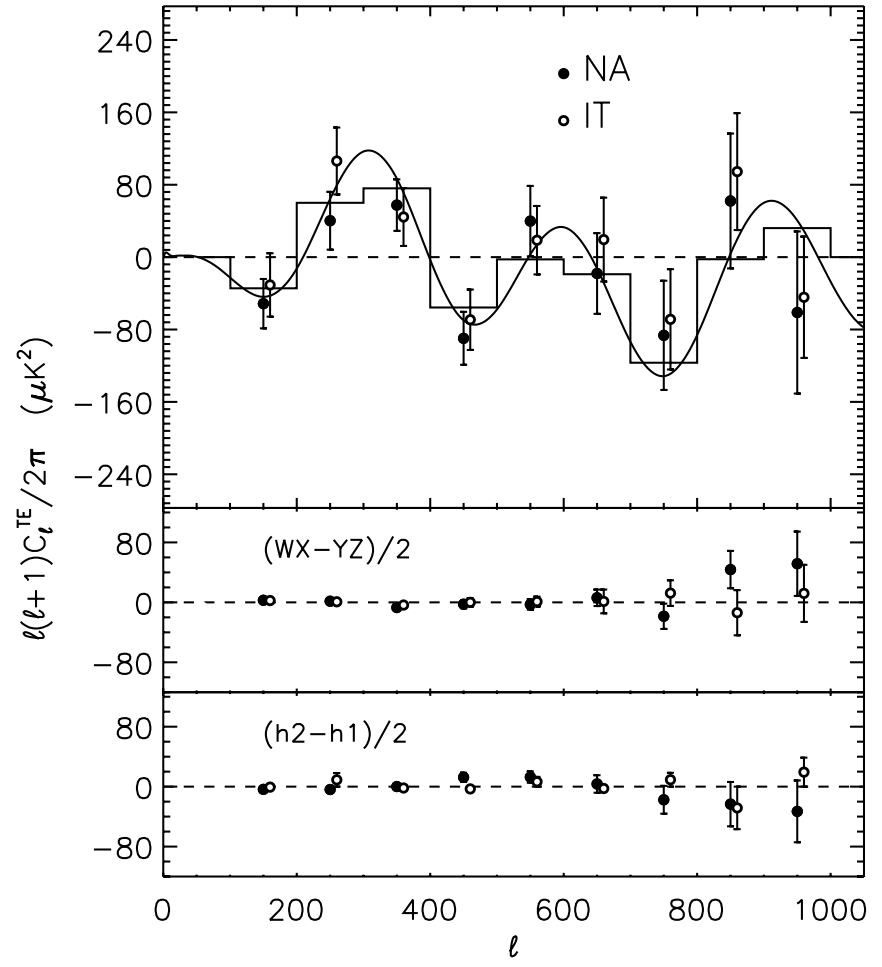

FIG. 1.-The $\langle$ TE $\rangle$ power spectrum band powers for the NA ( filled circles) and IT (open circles) pipelines. The upper part of the plot reports data with error bars, the fiducial model ( $\Lambda \mathrm{CDM}$ model fit to $W M A P$ [year 1], ACBAR, and CBI ) as a black curve, and the binned fiducial model as a histogram. The middle and bottom plots are the results of two different consistency tests, obtained splitting the data in channels ( WX-YZ) and in time (second half-first half), respectively. In the low- $l$ part of the plot the effect of a different weighting scheme between IT and NA is evident, while at large multipoles the result is dominated by the same instrumental performances. The error bars in the top part of the plot include sample variance from the signal; the bottom two plots they only include instrumental noise. [See the electronic edition of the Journal for a color version of this figure.]

$\langle\mathrm{TE}\rangle$ (but the same applies to $\langle\mathrm{TB}\rangle$ ), in the NA pipeline we perform independent analysis of the shallow scans and of the deep scans, computing the respective $a_{l m}^{T}$ and $a_{l m}^{E}$. We then estimate four $\langle\mathrm{TE}\rangle$ cross spectra, $\left\langle\mathrm{T}_{s} \mathrm{E}_{s}\right\rangle,\left\langle\mathrm{T}_{s} \mathrm{E}_{d}\right\rangle,\left\langle\mathrm{T}_{d} \mathrm{E}_{s}\right\rangle$, and $\left\langle\mathrm{T}_{d} \mathrm{E}_{d}\right\rangle$, with the respective correlation matrices and combine the spectra appropriately (C. R. Contaldi 2006, in preparation). In the IT pipeline, we combine the data in a single map with all the scans included. The $a_{l m}^{T}$ are computed on the shallow region, the $a_{l m}^{E}$ on the deep. The effect of such a double coverage is taken into account in the determination of the transfer function and the kernel used to derive the spectra.

\subsection{Result and Significance}

The results for the $\langle\mathrm{TE}\rangle$ and $\langle\mathrm{TB}\rangle$ power spectra are reported in the top panels of Figures 1 and 2, respectively, and in Table 1. The band powers cover a multipole range from $l=100$ to 1000 with nine bands 100 multipoles wide. In the process of power spectra estimation a low- and a high- $l$ band were included, but the power in these bands is not reported in the results. The low- $l$ bin is excluded because it includes mostly modes that are not constrained given our coverage and therefore have little meaning. The high- $l$ bin was excluded because it is the one most likely contaminated by the aliasing of all the excluded modes ( $l$ greater than the maximum multipole number included in the analysis). Both pipelines assume a flat shape for the power in each band (see eq. [4]). For comparison with model band powers, the IT pipeline assumes flat band power window functions, while the

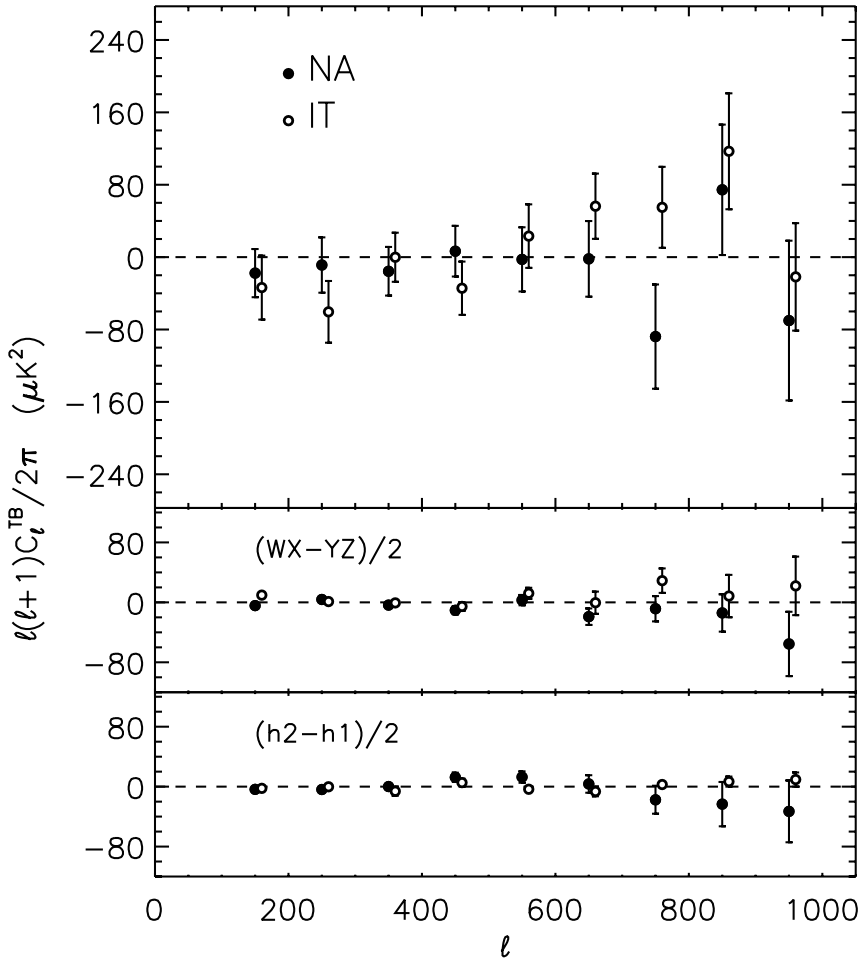

FIG. 2.-The $\langle\mathrm{TB}\rangle$ power spectrum band powers for the NA ( filled circles) and IT (open circles) pipelines. The upper part of the plot reports the $\langle\mathrm{TB}\rangle$ data with error bars. The middle and bottom plots are the results of two different consistency tests, obtained by splitting the data in channels ( WX-YZ) and in time (second halffirst half), respectively. The error bars in the top part of the plot include sample variance from the signal, while the bottom two plots only include instrumental noise. [See the electronic edition of the Journal for a color version of this figure.]

NA pipeline computes from the Xfaster Fisher matrix estimator the band power window functions $W_{l}^{b}$, that are used, in place of $P_{l}^{b}$, to convert a model power spectra $\mathcal{C}_{l}^{\text {mod }}=l(l+1) C_{l}^{\bmod } / 2 \pi$ into the theoretical band powers as

$$
\mathcal{C}_{b}^{\bmod }=\frac{\mathcal{I}\left(W_{l}^{b} \mathcal{C}_{l}^{\mathrm{mod}}\right)}{\mathcal{I}\left(W_{l}^{b}\right)}
$$

where $\mathcal{I}\left(f_{l}\right)=\sum_{l}\{(l+1 / 2) /[l(l+1)]\} f_{l}$ is the logarithmic integral defined in Bond et al. (2000). Band powers, covariance matrices, and window functions are available at the BOOMERANG Web sites. $^{21}$

To quantify the agreement of the detection with standard cosmology, we compare the data to $\mathcal{C}_{b, \Delta l}^{\text {mod }}$, the theoretical band powers of a fiducial model given by the $\Lambda$ CDM model of Spergel et al. (2003) fit to WMAP (year 1), Arcminute Cosmology Bolometer Array Receiver (ACBAR), and CBI, which we scale by an amplitude factor $a$ and shift by $\Delta l$ in multipole space. This phenomenological analysis is aimed to show the agreement between the polarization data and the concordance model as is common practice in the analysis of other data sets (see, e.g., Readhead et al. 2004). The simple shift in $l$ is motivated by the fact that, in the case of primordial adiabatic density fluctuations, given a $\langle\mathrm{TT}\rangle$ power spectrum, the $\langle\mathrm{TE}\rangle$ peaks are expected to have a $\pi / 2$ phase shift in $l$-space with respect to $\langle\mathrm{TT}\rangle$ peaks ( $\mathrm{Hu}$ $\&$ White 1997). For the physical interpretation, in terms of

21 See http://oberon.roma1.infn.it/boomerang/b2k and http://cmb.phys.cwru edu/boomerang. 
TABLE 1

BOOMERANG 2003 Polarization-Temperature Cross-Power Spectra Band Powers

\begin{tabular}{|c|c|c|c|c|c|c|c|c|}
\hline \multirow[b]{2}{*}{$l_{b}$} & \multicolumn{4}{|c|}{ NA } & \multicolumn{4}{|c|}{ IT } \\
\hline & $\begin{array}{c}\mathcal{C}_{b}^{\mathrm{TE}} \\
\left(\mu \mathrm{K}_{\mathrm{CMB}}^{2}\right)\end{array}$ & $\begin{array}{c}\Delta \mathcal{C}_{b}^{\mathrm{TE}} \\
\left(\mu \mathrm{K}_{\mathrm{CMB}}^{2}\right)\end{array}$ & $\begin{array}{c}\mathcal{C}_{b}^{\mathrm{TB}} \\
\left(\mu \mathrm{K}_{\mathrm{CMB}}^{2}\right)\end{array}$ & $\begin{array}{c}\Delta \mathcal{C}_{b}^{\mathrm{TB}} \\
\left(\mu \mathrm{K}_{\mathrm{CMB}}^{2}\right)\end{array}$ & $\begin{array}{c}\mathcal{C}_{b}^{\mathrm{TE}} \\
\left(\mu \mathrm{K}_{\mathrm{CMB}}^{2}\right)\end{array}$ & $\begin{array}{c}\Delta \mathcal{C}_{b}^{\mathrm{TE}} \\
\left(\mu \mathrm{K}_{\mathrm{CMB}}^{2}\right)\end{array}$ & $\begin{array}{c}\mathcal{C}_{b}^{\mathrm{TB}} \\
\left(\mu \mathrm{K}_{\mathrm{CMB}}^{2}\right)\end{array}$ & $\begin{array}{c}\Delta \mathcal{C}_{b}^{\mathrm{TB}} \\
\left(\mu \mathrm{K}_{\mathrm{CMB}}^{2}\right)\end{array}$ \\
\hline 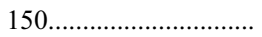 & -51 & 27 & -18 & 27 & -51 & 27 & -17 & 26 \\
\hline $250 \ldots \ldots \ldots \ldots \ldots \ldots \ldots \ldots$ & 40 & 32 & -9 & 31 & 40 & 31 & -8 & 30 \\
\hline 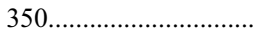 & 58 & 28 & -16 & 27 & 57 & 28 & -15 & 26 \\
\hline 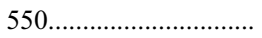 & 40 & 39 & -2 & 35 & 39 & 38 & -2 & 35 \\
\hline 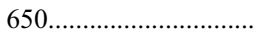 & -18 & 45 & -2 & 42 & -18 & 44 & -1 & 41 \\
\hline 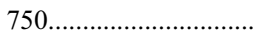 & -86 & 60 & -88 & 58 & -86 & 60 & -87 & 57 \\
\hline 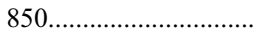 & 62 & 74 & 74 & 72 & 62 & 74 & 74 & 72 \\
\hline 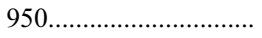 & -61 & 90 & -70 & 88 & -61 & 89 & -70 & 88 \\
\hline
\end{tabular}

Notes. - The uncertainties are given by the square root of the diagonal part of the covariance matrices. Bandwidth is $\Delta l=100$ for each bin. Complete results, including window functions and covariance matrices, are available at http://oberon.roma1.infn.it/boomerang/b2k and http://cmb.phys.cwru.edu/ boomerang.

cosmological parameters, see MacTavish et al. (2006). We compute the likelihood as

$$
L(a, \Delta l) \propto \exp \left[-\frac{1}{2} \sum_{b b^{\prime}}\left(\mathcal{C}_{b}-a \mathcal{C}_{b, \Delta l}^{\text {mod }}\right) \mathbf{M}_{b b^{\prime}}^{-1}\left(\mathcal{C}_{b^{\prime}}-a \mathcal{C}_{b^{\prime}, \Delta l}^{\bmod }\right)\right]
$$

where $\mathbf{M}_{b b^{\prime}}$ is the band powers' covariance matrix. Given the fact that the $\langle\mathrm{TE}\rangle$ power spectrum model crosses the zero several times, to improve the detection we used in this analysis a binning width of 50 multipole numbers and the corresponding covariance matrix.

The result is reported in Figure 3, together with the onedimensional likelihoods obtained by marginalization. For the $\langle\mathrm{TE}\rangle$ data, the likelihood defined as above favors a multipole shift in the range $-44<\Delta l<14$ for IT $(-38<\Delta l<20$ for NA) and an amplitude in the range $0.36<a<1.17$ for IT $(0.48<a<$ 1.38 for NA) at $95 \%$ probability. The data are thus in agreement with the amplitude of the $\langle\mathrm{TE}\rangle$ power spectrum predicted from the $\langle\mathrm{TT}\rangle$ power spectrum under the hypothesis of adiabatic initial perturbations. For the $\langle\mathrm{TB}\rangle$ data, the likelihood (with respect to a $\langle\mathrm{TE}\rangle$ fiducial model) do not constrain the multipole shift and gives an amplitude in the range $-0.51<a<0.23$ for IT $(-0.38<a<0.47$ for NA) at $95 \%$ of probability.

To compare our data to a model $\mathcal{H}_{0}$ characterized by a parameter set $\boldsymbol{p}$, we define the goodness of fit of the model as

$$
\Lambda(\boldsymbol{p})=\log \left[\frac{L\left(\boldsymbol{p}_{\mathrm{ML}}\right)}{L(\boldsymbol{p})}\right]
$$

where $L\left(\boldsymbol{p}_{\mathrm{ML}}\right)$ and $L(\boldsymbol{p})$ are the values of the likelihood at the maximum and for the parameters $\boldsymbol{p}$ of the model, respectively. In the approximation that the likelihood function $L(\boldsymbol{p})$ is a multivariate Gaussian near its peak, the goodness of fit reduces to $\Lambda=\Delta \chi^{2} / 2$, and the probability of total exclusion is defined by the incomplete gamma function

$$
\operatorname{PTE}(\Lambda)=\frac{1}{\Gamma(N / 2)} \int_{\Lambda}^{\infty} e^{-x} x^{N / 2-1} d x
$$

where $N$ is the number of parameters in the model, which is 2 , the amplitude $a$ and the shift $\Delta l$, in our case. A set of tests performed using 19 bins between $l=100$ and 1000 is reported in Table 2 .
In that table, $\sigma$ represents the number of standard deviations of a normal distribution to have the same PTE as $\Lambda$ does. The $\langle\mathrm{TE}\rangle=$ 0 model is rejected at $3.5 \sigma$ for both pipelines, and the $\langle\mathrm{TB}\rangle=$ $\langle\mathrm{TE}\rangle^{\mathrm{mod}}$ is rejected at $4.0 \sigma$ for NA and $6.1 \sigma$ for IT.

\section{CONTROL OF FOREGROUNDS}

Polarization generated by foregrounds presents no global symmetry and thus is expected to contaminate both $E$ - and $B$-components of the CMB in a similar way (see, e.g., Tegmark

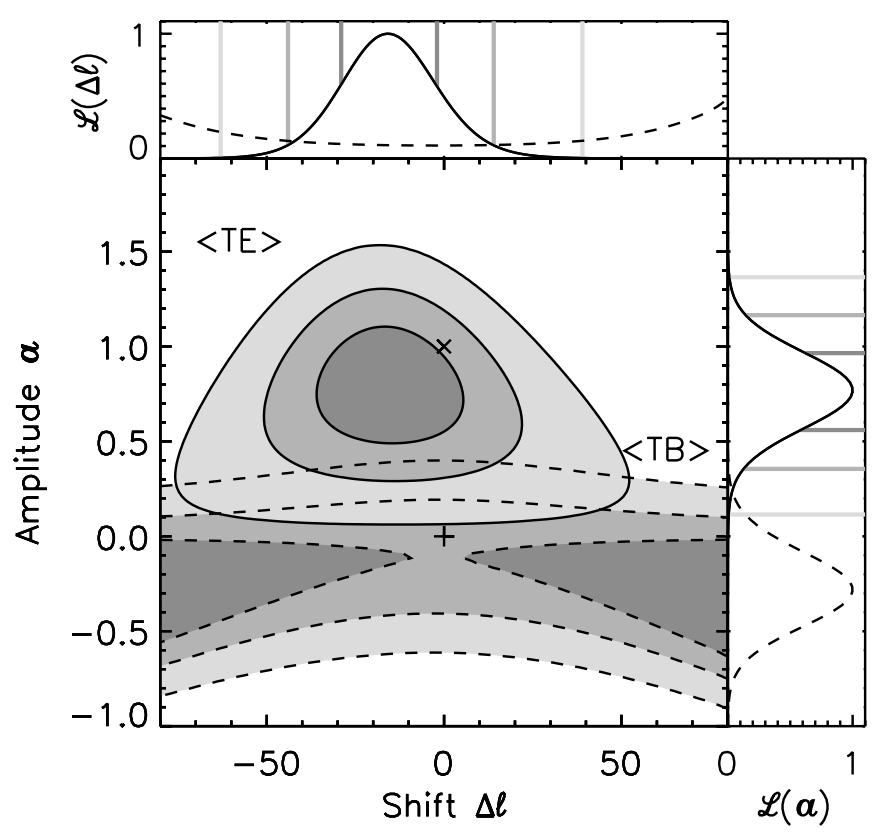

FIG. 3.-Likelihood of the parameters $a$ and $\Delta l$. Parameter $a$ is defined as the amplitude of the $\langle\mathrm{TE}\rangle$ fiducial model, and $\Delta l$ is the shift in multipole $l$ applied to the $\langle\mathrm{TE}\rangle$ fiducial model. The continuum lines are for the BOOMERANG $\langle\mathrm{TE}\rangle$ data; the dashed lines are for $\langle\mathrm{TB}\rangle$ data, compared to the same $\langle\mathrm{TE}\rangle$ fiducial model. In the central plot the two-dimensional likelihood, $L(\Delta l, a)$ is reported; the contours are 1,2 , and $3 \sigma$, corresponding to $68.3 \%, 95.4 \%$, and $99.7 \%$ probability, respectively. The cross is the expected value for $\langle\mathrm{TE}\rangle$ given the fiducial model; the plus sign is the expected value for $\langle\mathrm{TB}\rangle$. In the right plot the $L(a)$ marginalizing over $\Delta l$ is reported, and in the top plot, the $L(\Delta l)$ marginalizing over $a$. In the right and top plot, the gray lines are 1,2, and $3 \sigma$ boundaries for the $\langle\mathrm{TE}\rangle$ data. The $\langle\mathrm{TB}\rangle$ data likelihood is used to test the presence of foregrounds and systematic effects that would affect $\langle\mathrm{TE}\rangle$ and $\langle\mathrm{TB}\rangle$ in the same way. This plot is obtained using the IT data set with a binning width of 50 multipole numbers. The NA data set gives a similar result. 
TABLE 2

$\langle\mathrm{TE}\rangle$ and $\langle\mathrm{TB}\rangle$ Statistics

\begin{tabular}{|c|c|c|c|c|c|c|}
\hline Pipeline & Test & dof & $\chi^{2}$ & $\Lambda$ & PTE & $\sigma_{\text {reject. }}$ \\
\hline \multirow[t]{4}{*}{ 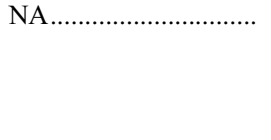 } & $\langle\mathrm{TE}\rangle$ compared to fiducial & 9 & 5.81 & 0.48 & 0.61 & \\
\hline & $\langle\mathrm{TE}\rangle$ compared to zero & 9 & 23.1 & 8.23 & $2.6 \times 10^{-4}$ & 3.5 \\
\hline & $\langle\mathrm{TB}\rangle$ compared to zero & 9 & 4.94 & 0.08 & 0.92 & \\
\hline & $\langle\mathrm{TB}\rangle$ compared to fiducial $\langle\mathrm{TE}\rangle$ & 9 & 10.5 & 10.0 & $2.9 \times 10^{-5}$ & 4.0 \\
\hline \multirow[t]{4}{*}{ IT ............... } & $\langle\mathrm{TE}\rangle$ compared to fiducial & 9 & 8.00 & 1.65 & 0.19 & \\
\hline & $\langle\mathrm{TE}\rangle$ compared to zero & 9 & 19.7 & 8.20 & $2.7 \times 10^{-4}$ & 3.5 \\
\hline & $\langle\mathrm{TB}\rangle$ compared to zero & 9 & 13.3 & 2.44 & 0.09 & \\
\hline & $\langle\mathrm{TB}\rangle$ compared to fiducial $\langle\mathrm{TE}\rangle$ & 9 & 44.1 & 21.2 & $5.8 \times 10^{-10}$ & 6.1 \\
\hline
\end{tabular}

Note.-Significance of the $\langle\mathrm{TE}\rangle$ and $\langle\mathrm{TB}\rangle$ results with respect to models.

et al. 2000; Tucci et al. 2002; Baccigalupi et al. 2001). The $\langle$ TB $\rangle$ power spectrum can be used to test such a contamination. The $\langle\mathrm{TB}\rangle=0$ result presented in Table 2 is the main evidence that the $\langle\mathrm{TE}\rangle$ result is not contaminated.

Moreover, we can directly test the contamination due to dust by a correlation of our data with a dust map (see, e.g., Ponthieu et al. 2005). If we assume that the temperature and polarization seen by BOOMERANG are a superposition of CMB and dust, $T_{\mathrm{B} 03}=$ $T_{\mathrm{CMB}}+T_{\text {dust }}$ and $E_{\mathrm{B} 03}=E_{\mathrm{CMB}}+E_{\text {dust }}($ and the same for $B$ ), then

$$
\langle\mathrm{TE}\rangle_{\mathrm{B} 03}=\left\langle\mathrm{T}_{\mathrm{CMB}} \mathrm{E}_{\mathrm{CMB}}\right\rangle+\left\langle\mathrm{T}_{\text {dust }} \mathrm{E}_{\text {dust }}\right\rangle,
$$

where we assume that dust and $\mathrm{CMB}$ are not correlated $\left(\left\langle\mathrm{T}_{\mathrm{CMB}} \mathrm{E}_{\text {dust }}\right\rangle=\left\langle\mathrm{T}_{\text {dust }} \mathrm{E}_{\mathrm{CMB}}\right\rangle=0\right)$. Under the same assumption, the contaminating term $\left\langle\mathrm{T}_{\text {dust }} \mathrm{E}_{\text {dust }}\right\rangle$ can be estimated by

$$
\left\langle\mathrm{T}_{\text {dust }} \mathrm{E}_{\text {dust }}\right\rangle \simeq\left\langle\mathrm{T}_{\text {dust }} \mathrm{E}_{\mathrm{B} 03}\right\rangle
$$

We estimate $T_{\text {dust }}$ by using Infrared Astronomical Satellite (IRAS) maps recalibrated with Diffuse Infrared Background Experiment (DIRBE) at $100 \mu \mathrm{m}$, extrapolated to our wavelength with model 8 in Finkbeiner et al. (1999), as described in Masi et al. (2001). We resample the extrapolated dust map with the BOOMERANG scan strategy and then recreate a dust map with the same time domain filtering, flagging, and mapmaking algorithm as the

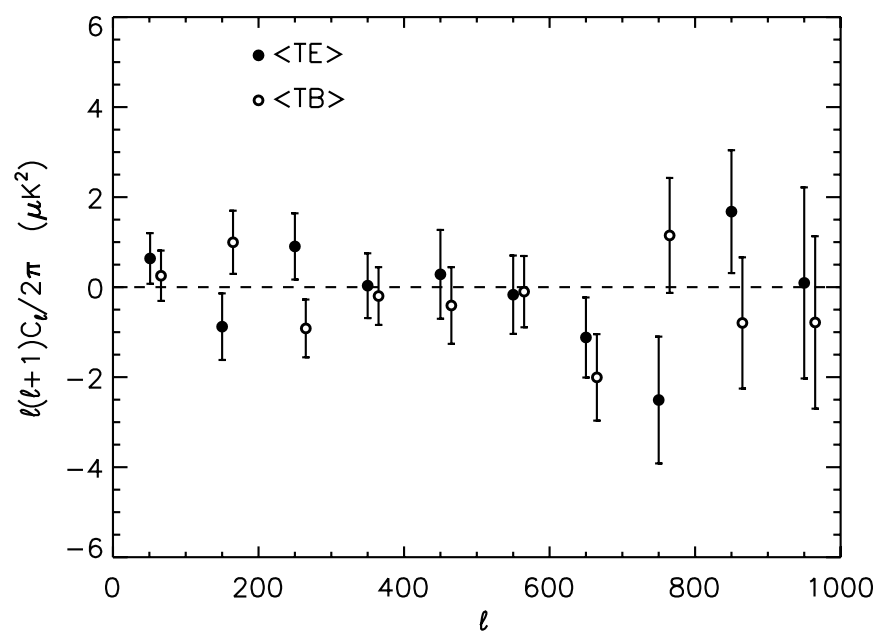

Fig. 4.-Dust contamination. Filled circles are $\left\langle\mathrm{T}_{\mathrm{dust}} \mathrm{E}_{\mathrm{B} 03}\right\rangle$; open circles are $\left\langle\mathrm{T}_{\text {dust }} \mathrm{B}_{\mathrm{B} 03}\right\rangle$. The dust contamination to $\langle\mathrm{TE}\rangle$ is 2 orders of magnitude lower than the measured $\langle\mathrm{TE}\rangle$. The B03 data are from the IT pipeline. [See the electronic edition of the Journal for a color version of this figure.]
BOOMERANG map. To estimate the error bars, we calculate the same $\langle\mathrm{TE}\rangle$ as in equation (12) for a set of Monte Carlo simulations of CMB signal and BOOMERANG noise, using the same weighting scheme. Then we use the rms in each bin as the value of the uncertainty. In this way, we also take into account random alignment of dust temperature to $\mathrm{CMB}$ polarization and instrumental noise projected into the map. As shown in Figure 4, this contaminant is compatible with zero and is 2 orders of magnitude lower than the measured $\langle\mathrm{TE}\rangle$.

\section{CONTROL OF SYSTEMATIC EFFECTS}

The standard test to detect systematic effects consists of splitting the data in two subsets, making a differenced map from the two subsets and calculating the power spectra of the differenced map, divided by two to maintain the same noise statistics as an average map. The result must be consistent with zero. This test is particularly effective because the sample variance goes to zero and the noise in equation (6) reduces to

$$
\sigma_{\mathrm{TE}, b}^{2}=\frac{2}{\left(2 l_{b}+1\right) f_{\mathrm{sky}} \Delta l}\left(\frac{N_{T, b} N_{E, b}}{B_{b}^{4}}\right) .
$$

We performed two such tests, splitting the data in time and in channels. As our temporal splitting we take the first half of the scans on the shallow region and the first half of the scans on the deep region versus the second half of shallow plus the second half of deep. As our channel splitting, we take two PSB pairs (W1, W2, X1, X2) versus the other two PSB pairs ( Y1, Y2, Z1, Z2; for focal plane description, see Masi et al. [2006]). Results are reported in the bottom panels of Figures 1 and 2 and in Table 3, showing remarkable consistency with zero.

These tests can dilute scan-synchronous noise, which is instead detectable in the time stream. To check the effect of that, we

\begin{tabular}{|c|c|c|c|c|}
\hline Pipeline & Test & dof & $\chi^{2}$ & $P_{>}$ \\
\hline \multirow[t]{4}{*}{ NA } & $\langle\mathrm{TE}\rangle$ temporal & 9 & 16.1 & 0.065 \\
\hline & $\langle\mathrm{TE}\rangle$ channels & 9 & 10.6 & 0.30 \\
\hline & $\langle\mathrm{TB}\rangle$ temporal & 9 & 13.1 & 0.16 \\
\hline & $\langle\mathrm{TB}\rangle$ channels & 9 & 12.3 & 0.20 \\
\hline \multirow[t]{4}{*}{ IT } & $\langle\mathrm{TE}\rangle$ temporal & 9 & 14.8 & 0.10 \\
\hline & $\langle\mathrm{TE}\rangle$ channels & 9 & 2.42 & 0.98 \\
\hline & $\langle\mathrm{TE}\rangle$ temporal & 9 & 7.55 & 0.58 \\
\hline & $\langle\mathrm{TB}\rangle$ channels & 9 & 13.5 & 0.14 \\
\hline
\end{tabular}

TABLE 3

Consistency Tests 


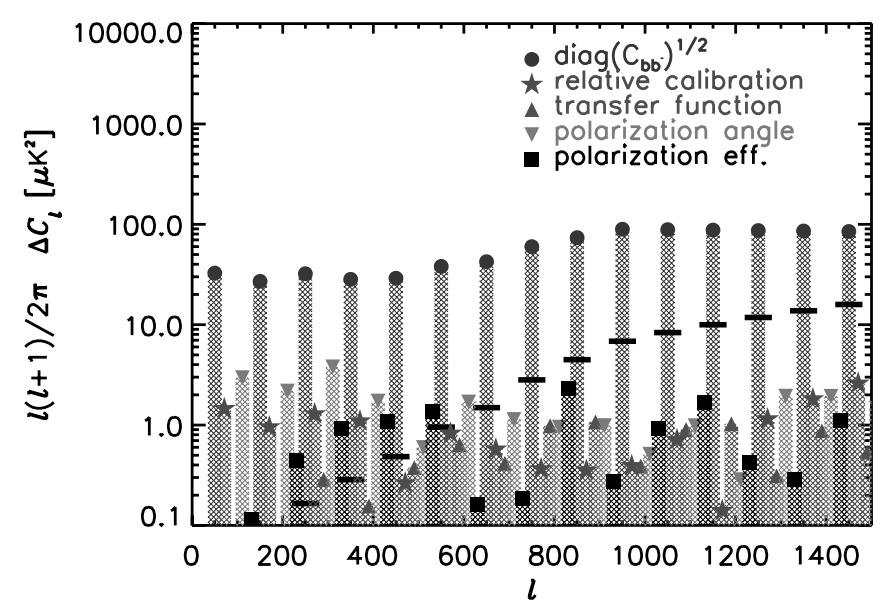

FIG. 5.-Propagation of instrumental uncertainties in the $\langle\mathrm{TE}\rangle$ error bars. The dots are the square root of the diagonal part of the covariance matrix and represent the statistical uncertainty on the result. Relative calibration is varied by $\pm 0.4 \%$, polarization efficiency by \pm 0.03 , time constants of the transfer function by $\pm 10 \%$, the angles of the polarizers respect to the telescope frame by $\pm 2^{\circ}$, and the beam (horizontal thick lines) by \pm 0.3 . The effect of scan-synchronous noise, which was also tested, is below $0.1 \mu \mathrm{K}^{2}$. The error bars $\left(\Delta C_{l}\right)$ generated by uncertainties in instrumental characteristics are 1 order of magnitude lower than the errors due to noise and sampling variance (from NA pipeline here). Those error bars are not treated as an increased error, but rather as a systematic effect, which is correlated bin-to-bin, and marginalized over in the parameter estimation as described in Bridle et al. (2002). [See the electronic edition of the Journal for a color version of this figure.]

simulate a scan-synchronous time stream filtered and calibrated with the proper factors, and run it through the pipeline. The resulting $\langle\mathrm{TE}\rangle$ power spectrum is below $0.1 \mu \mathrm{K}^{2}$ at all multipoles and thus is negligible respect to the error bars of our data. These tests, along with the $\langle\mathrm{TB}\rangle=0$ result presented above, give strong evidence that the data set is free from significant systematics.

\subsection{Propagation of Instrumental Uncertainties}

In addition, we have modeled the potential systematic effects from misestimation of various instrumental parameters using Monte Carlo simulations of signal-only time-ordered data, processed varying those parameters randomly over their range of uncertainty with a Gaussian distribution. The parameters that have been changed are, the relative calibration between channels $( \pm 0.4 \%)$, the polarization efficiency $( \pm 0.03)$, the bolometer time constants $( \pm 10 \%)$, the beam $( \pm 0.3)$, and the angles of the polarizer's axes respect to the telescope frame $\left( \pm 2^{\circ}\right)$. These ranges are the uncertainties on those instrumental parameters as described in Masi et al. (2006).

As shown in Figure 5, the potential errors from misestimation of instrumental parameters are all at least 1 order of magnitude lower than the statistical error bars of the data set. The simulation uses the $\Lambda C D M$ model of Spergel et al. (2003) fit to $W M A P$ (year 1), ACBAR, and CBI.

\section{CONCLUSION}

We have detected the presence of polarization of the CMB with high statistical significance (3.5 $\sigma$ combining the bins). This detection of $\langle\mathrm{TE}\rangle$ confirms and improves previous lower frequency detections (Kogut et al. 2003; Leitch et al. 2005) using a completely independent technology. The robustness of these results against foreground contamination effects is thus strengthened, and

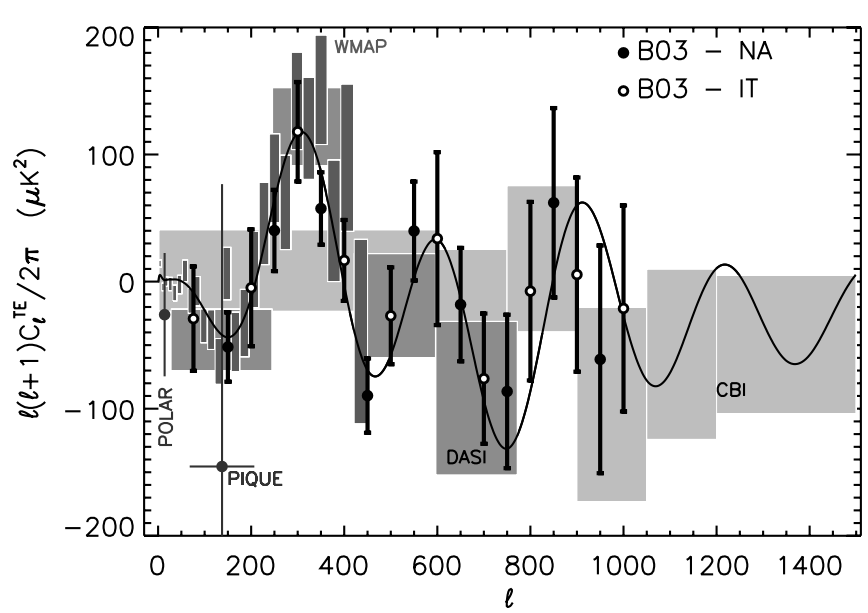

FIG. 6.-Collection of results for $\langle\mathrm{TE}\rangle$ power spectrum from recent experiments. The BOOMERANG data are NA and IT, with alternate binning. WMAP data are from Kogut et al. (2003), CBI from Readhead et al. (2004), DASI from Leitch et al. (2005), PIQUE from de Oliveira-Costa et al. (2003b), and POLAR from de Oliveira-Costa et al. (2003a). [See the electronic edition of the Journal for a color version of this figure.]

its cosmological origin confirmed. A summary of all measurements of the $\langle\mathrm{TE}\rangle$ spectrum is shown in Figure 6.

The B03 $\langle\mathrm{TE}\rangle$ data show a $2 \sigma$ anticorrelation at large angular scales $(50<l<150)$. This, as previously detected by the WMAP experiment (Peiris et al. 2003), is consistent with the presence of superhorizon adiabatic fluctuations (Spergel \& Zaldarriaga 1997) and does not support models based on active perturbations like topological defects (Turok 1996). In active models the perturbations are continuously produced by the causal field and lead to a positive correlation in the $\langle\mathrm{TE}\rangle$ spectrum. While cosmic string and textures models are already ruled out by the presence of peaks in the CMB temperature power spectrum, active models may be constructed (see e.g., Durrer et al. 2001) to mimic the $\langle\mathrm{TT}\rangle$ data but not the $\langle\mathrm{TE}\rangle$ spectrum.

We gratefully acknowledge support from CIAR, CSA, and NSERC in Canada, ASI, University La Sapienza and PNRA in Italy, PPARC and the Leverhulme Trust in the UK, and NASA (awards NAG5-9251 and NAG5-12723) and NSF (awards OPP 99-80654 and OPP 04-07592) in the US. Additional support for detector development was provided by CIT and the Jet Propulsion Laboratory (JPL). C. B. N. acknowledges support from a Sloan Foundation Fellowship; W. C. J. and T. E. M. were partially supported by NASA GSRP Fellowships. Field, logistical, and flight support was outstandingly supplied by USAP and NSBF; data recovery was especially appreciated. This research used resources at NERSC, supported by the DOE under contract DEAC03-76SF00098, and the MacKenzie cluster at CITA, funded by the Canada Foundation for Innovation. We also thank the CASPUR (Rome, Italy) computational facilities and the Applied Cluster Computing Technologies Group at the JPL for computing time and technical support. Some of the results in this paper have been derived using the HEALPix (Górski et al. 2005) package, and nearly all have benefited from the FFTW3 implementation of fast Fourier transform (Frigo \& Johnson 2005). We acknowledge the referee for useful comments. The BOOMERANG field team is also grateful to the Coffee House at McMurdo Station, Antarctica, for existing. 
REFERENCES

Baccigalupi, C., Burigana, C., Perrotta, F., De Zotti, G., La Porta, L., Maino, D., Maris, M., \& Paladini, R. 2001, A\&A, 372, 8

Bond, J. R., \& Efstathiou, G. 1984, ApJ, 285, L45

Bond, J. R., Jaffe, A. H., \& Knox, L. 2000, ApJ, 533, 19

Bridle, S. L., Crittenden, R., Melchiorri, A., Hobson, M. P., Kneissl, R., \& Lasenby, A. N. 2002, MNRAS, 335, 1193

Bucher, M., Moodley, K., \& Turok, N. 2001, Phys. Rev. Lett., 87, 191301

Coulson, D., Crittenden, R. G., \& Turok, N. G. 1994, Phys. Rev. Lett., 73, 2390

Crittenden, R. G., Coulson, D., \& Turok, N. G. 1995, Phys. Rev. D, 52, 5402

de Oliveira-Costa, A., Tegmark, M., O'Dell, C., Keating, B., Timbie, P.,

Efstathiou, G., \& Smoot, G. 2003a, Phys. Rev. D, 68, 083003

de Oliveira-Costa, A., Tegmark, M., Zaldarriaga, M., Barkats, D., Gundersen,

J. O., Hedman, M. M., Staggs, S. T., \& Winstein, B. 2003b, Phys. Rev. D, 67, 023003

Durrer, R., Kunz, M., \& Melchiorri, A. 2001, Phys. Rev. D, 63, 081301

Efstathiou, G. P. 1988, in Large-Scale Motions in the Universe, ed. V. Rubin \& S. J. Coyne (Princeton: Princeton Univ. Press), 299

Finkbeiner, D. P., Davis, M., \& Schlegel, D. J. 1999, ApJ, 524, 867

Frigo, M., \& Johnson, S. G. 2005, Proc. IEEE, 93, 216

Górski, K. M., Hivon, E., Banday, A. J., Wandelt, B. D., Hansen, F. K., Reinecke, M., \& Bartelmann, M. 2005, ApJ, 622, 759

Hivon, E., Górski, K. M., Netterfield, C. B., Crill, B. P., Prunet, S., \& Hansen, F. 2002, ApJ, 567, 2

Hu, W., \& White, M. 1997, NewA, 2, 323

Jones, W. C., et al. 2006, ApJ, 647, 823

Kamionkowski, M., Kosowsky, A., \& Stebbins, A. 1997, Phys. Rev. Lett., 78, 2058
Kogut, A., et al. 2003, ApJS, 148, 161

Kosowsky, A. 1999, NewA Rev., 43, 157

Kovac, J. M., Leitch, E. M., Pryke, C., Carlstrom, J. E., Halverson, N. W., \& Holzapfel, W. L. 2002, Nature, 420, 772

Leitch, E. M., Kovac, J. M., Halverson, N. W., Carlstrom, J. E., Pryke, C., \& Smith, M. W. E. 2005, ApJ, 624, 10

MacTavish, C. J., et al. 2006, ApJ, 647, 799

Masi, S., et al. 2001, ApJ, 553, L93 2006, A\&A, submitted

Montroy, T. E., et al. 2006, ApJ, 647, 813

Peiris, H. V., et al. 2003, ApJS, 148, 213

Pogosian, L., Vachaspati, T., \& Winitzki, S. 2002, Phys. Rev. D, 65, 083502

Ponthieu, N., et al. 2005, A\&A, 444, 372

Readhead, A. C. S., et al. 2004, Science, 306, 836

Rees, M. J. 1968, ApJ, 153, L1

Seljak, U., \& Zaldarriaga, M. 1996, ApJ, 469, 437

Spergel, D. N., \& Zaldarriaga, M. 1997, Phys. Rev. Lett., 79, 2180

Spergel, D. N., et al. 2003, ApJS, 148, 175

Tegmark, M., Eisenstein, D. J., Hu, W., \& de Oliveira-Costa, A. 2000, ApJ, 530,133

Tucci, M., Carretti, E., Cecchini, S., Nicastro, L., Fabbri, R., Gaensler, B. M., Dickey, J. M., \& McClure-Griffiths, N. M. 2002, ApJ, 579, 607

Turok, N. 1996, Phys. Rev. Lett., 77, 4138

Zaldarriaga, M. 1997, Phys. Rev. D, 55, 1822

Zaldarriaga, M., \& Seljak, U. 1997, Phys. Rev. D, 55, 1830 